\title{
Rethinking public mental health: learning from Models of Obesity
}

Stanley J. Ulijaszek's new book Models of Obesity (published October 2017) may be thought to be primarily of interest to obesity researchers, but in fact offers potential applications beyond obesity, in the arena of public mental health. ${ }^{1}$ Parallels between public health responses to obesity and mental health issues are already apparent; in both cases concern about rising levels have come increasingly to the fore; what constitutes normality is contested; how far problems are socially constructed or appropriately medicalised is debated; and there is an increasing focus on children as a site of intervention. But there are also parallels which, while less immediately obvious, might be of crucial importance in understanding and advancing the practice of public mental health.

First, the chronic relapsing nature of the condition needs to be recognised. There is an urgent need to reframe mental health problems, as has been done for obesity, as "chronic relapsing" conditions. Currently if you ask mental health practitioners if mental health issues are acute or chronic they shift uncomfortably and answer: "both" or "it depends". Until we determine how to distinguish acute from chronic mental health issues, from a public health point of view mental health issues should be conceived as chronic relapsing-remitting. ${ }^{2,3}$

Second, agreeing a standard metric of measurement is key to advancing the field. Ulijaszek notes that "The standardization of obesity measurement using BMI has allowed sense to be made of population obesity more easily through the practice of epidemiology. ${ }^{11}$ Entrenched confusion about terminology acts as a key barrier to achieving this goal in mental health, but it is vital we do so. Terms such as mental illness, mental health problems, and mental distress are used interchangeably; "mental health" and "wellbeing" are inappropriately lumped together, as if they are one construct. 4,5 Some consensus on use of self-report on standardised measures is emerging but needs to agree focus and thresholds. ${ }^{3,6}$

Third, researchers and practitioners need to consider complex systems that contribute to the public health problem. Ulijaszek points out that whilst obesity (like mental health) is multidetermined, there are aspects of the environment that can be seen as "obesogenic": "Obesogenic environments are easily identified by a great preponderance of motorized transport and of sedentary occupations, and the cheap and easy availability of high-fat, high-refined-carbohydrate foods." ${ }^{1}$ Some aspects of what we might term "psychopathogenic" environments are clear; environments with high levels of abuse, poverty, bullying, and social isolation. ${ }^{7}$ Other aspects of psychopathogenic environments such as the possible impact of social media, work and educational pressures, and lack of exercise or adequate sleep are more open to debate and await further research.

Fourth, the need exists for a "poly rational" approach drawing on a range of evidences. Ulijaszek states that obesity public health is "situated in a field of competing interests and behaviours...The corporations, agencies, governments and institutions that form components of these systems act using a range of different rationalities, incompatibilities in which may contribute to obesity production. Poly rational approaches...would require understanding these rationalities, their relationships to each other and the extent to which they predispose to obesity. ${ }^{1}$ Recent funding initiatives in mental health (e.g., the ESRC-led network call in the UK) attempt to bring together different "rationalities" but the need to consider how best to do this and to find a way to 
consider how far existing rationalities contribute to the problem needs further consideration. ${ }^{8}$ Ulijaszek notes "cacophony of effort" in addressing obesity, ${ }^{1,9}$ and to rationalise this Swinburn and colleagues ${ }^{10}$ propose a framework which groups evidence into non-hierarchical categories of: observational, experimental, extrapolated, and experience-based. In mental health, experimental studies are at the top of a hierarchy. Given the current state of evidence and the position of interventions within complex systems, extrapolated evidence (indirect or strongly assumed based on a range of findings from a variety of disciplines) may present the most productive focus for our attention currently. ${ }^{11}$

Finally, the research community should aim to find what Ulijaszek defines as "viable clumsy solutions", which are "composed of a combination of singly rational policies towards a particular problem". ${ }^{1}$ In order to agree such viable clumsy solutions those working to address obesity have developed structured approaches ${ }^{12}$ which bring stakeholders together to describe and analyse obesogenic environments in terms of physical, economic, policy, and socio-cultural environmental factors, and to explore options as to how to address these. Public mental health could draw on this approach. Key stakeholders might include those currently accessing mental health services as well as those managing difficulties without, or following, access, alongside the range of health, education, social care, and research agencies that might be seen as forming part of the complex system into which any public mental health initiative would be situated, including state, voluntary, and private organisations.

In conclusion, if key stakeholders can come together, with a shared understanding of mental health issues as a chronic relapsing public health concern, to map out psychopathogenic environments; consider what might be extrapolated from existing evidences; and agree a set of viable clumsy viable solutions that could be trialled alongside agreed metrics we might be on the way to substantial progress in public mental health. Potential barriers to this may be principally cultural. To move away from the current dedicated focus on increasing access to professionally-led individualised interventions drawing on solely bio-medical evidence, and to move towards outlining, analysing and addressing "psychopathogenic environments", drawing evidence from different disciplines, requires bringing together new networks of stakeholders. Some funders seem ready for this; the question is whether the stakeholders in our complex system are prepared for it.

Miranda Wolpert, Professor of Evidence Research \& Practice, UCL; Director of Evidence Based Practice Unit, UCL \& Anna Freud National Centre for Children \& Families and Director of Child Outcomes Research Consortium. All views are author's own and do not represent any of the organisations Miranda works for. Miranda.wolpert@ucl.ac.uk 


\section{$\underline{\text { References }}$}

1. Ulijaszek, S. J. (2017). Models of Obesity: From Ecology to Complexity in Science and Policy (Vol. 78). Cambridge University Press.

2. Whiteford, H. A., Harris, M. G., McKeon, G., Baxter, A., Pennell, C., Barendregt, J. J., \& Wang, J. (2013). Estimating remission from untreated major depression: a systematic review and meta-analysis. Psychological medicine, 43(8), 1569-1585.

3. Wolpert, M., Jacob, J., Napoleone, E., Whale, A., Calderon, A., \& Edbrooke-Childs, J. (2016). Child- and Parent-reported Outcomes and Experience from Child and Young People's Mental Health Services 2011-2015. London: CAMHS Press.

4. Sharpe, H., Patalay, P., Fink, E., Vostanis, P., Deighton, J., \& Wolpert, M. (2016). Exploring the relationship between quality of life and mental health problems in children: implications for measurement and practice. European child \& adolescent psychiatry, 25(6), 659-667.

5. Patalay, P., \& Fitzsimons, E. (2016). Correlates of mental illness and wellbeing in children: are they the same? Results from the UK Millennium Cohort Study. Journal of the American Academy of Child \& Adolescent Psychiatry, 55(9), 771-783.

6. Clark, D. M., Canvin, L., Green, J., Layard, R., Pilling, S., \& Janecka, M. (2017). Transparency about the outcomes of mental health services (IAPT approach): an analysis of public data. The Lancet.

7. Rutter, M. (2013). Annual research review: resilience-clinical implications. Journal of child psychology and psychiatry, 54(4), 474-487.

8. Furedi, F. (2004). Therapy culture: Cultivating vulnerability in an uncertain age. Psychology Press.

9. Lang, T. \& Rayner, G. (2007). Overcoming policy cacophony on obesity: an ecological public health framework for policymakers. Obesity Reviews 8 (Suppl. 1): 165-81.

10. Swinburn, B.A., Gill, T. \& Kumanyika, S. (2005). Obesity prevention: a proposed framework for translating evidence into action. Obesity Reviews 6: 23-33.

11. Rutter, Harry, Natalie Savona, Ketevan Glonti, Jo Bibby, Steven Cummins, Diane T. Finegood, Felix Greaves et al. "The need for a complex systems model of evidence for public health." Lancet (2017).

12. Swinburn, B.A., Egger, G. \& Raza, F. (1999). Dissecting obesogenic environments: the development and application of a framework for identifying and prioritizing environmental interventions for obesity. Preventive Medicine 29: 563-70. 